\title{
Hombres y mujeres en Sonora
}

\author{
Irma Laura Murillo Lozoya*
}

\section{Resumen}

El presente artículo aborda los diversos temas sobre la equidad de género, a la luz de las estadísticas existentes. La información disponible en la actualidad es amplia, e incluye aspectos como la educación, economía, salud, aspectos sociales y conyugales, la distribución de las tareas domésticas y la violencia hacia las mujeres, entre otros.

En este trabajo se abordan las diferencias históricas en las que se ha ido avanzando, hasta lograr que el acceso y el nivel de escolaridad de mujeres y hombres sea prácticamente el mismo; asimismo, que las tareas domésticas, aunque ligeramente, ya muestren una mayor participación por parte de los hombres, y que la esperanza de vida para las mujeres siga siendo relativamente más alta.

Entre los temas pendientes se encuentran lograr mejores oportunidades laborales para las mujeres y erradicar toda forma de violencia hacia ellas.

\footnotetext{
Abstract

This article presents various topics about gender equity considering the existing statistics. The information currently available is extensive, and includes aspects such as education, economy, health, social and marital issues, the distribution of housework and violence against women, among others.

In this paper, the historical differences have been eased, to get access and the level of education of women and men is almost the same, as well as greater participation of men in housework, and life expectancy for women is maintained relatively high.
}

Among the outstanding issues are achieve the best employment opportunities for women and eradicate all forms of violence against them.

\section{Introducción}

Hasta hace algunos años, la información con enfoque de género era escasa. Solo se disponía de pocas variables, de tal forma que realizar tareas de planeación basadas en información confiable era materialmente imposible.

En el transcurso del tiempo, la necesidad de información y la relevancia que en nuestro país y en el mundo han tomado los diversos temas en materia de equidad, llevaron a la disposición de mayores y mejores datos, que sin duda alguna son un gran apoyo para el análisis de los diversos fenómenos que tienen que ver con las diferencias entre mujeres y hombres.

La disponibilidad de la información constituye pues, una gran herramienta para que las y los investigadores y diseñadores de las políticas públicas, respalden con datos duros las medidas que implementen para mejorar y evaluar la situación en materia de equidad en nuestro país y nuestro estado.

\footnotetext{
* Licenciada en Economía por la Universidad Autónoma de Nuevo León, Diplomada en Demografía por el Centro Latinoamericano de Demografía de San José, Costa Rica. Directora Regional Noroeste en el Instituto Nacional de Estadística y Geografía. irma.murillo@inegi. org.mx
}

\section{Savìa}




\section{Características generales}

Iniciaremos por analizar la relación hombres-mujeres. Es muy común escuchar el dicho popular: "Para cada hombre hay siete mujeres". Sin embargo, no hay nada más fuera de la realidad.

En el país, la relación es de 95 hombres por cada 100 mujeres. Sonora es uno de los cuatro estados del país en los cuales hay más hombres que mujeres, aunque la diferencia sea mínima. Un aspecto que influye en este dato para nuestra entidad es la migración, ya que las actividades que atraen población por motivos de trabajo, como la minería y la agricultura, se caracterizan por captar mayoritariamente población masculina.

Lo anterior no es un fenómeno reciente, si observamos la información a lo largo de la historia, esta tendencia se ha mantenido permanentemente en nuestro estado.

En contraste, en el ámbito nacional y para algunas entidades de la república, la migración internacional, que es mayoritariamente de hombres, incide fuertemente en que haya una mayor cantidad de mujeres tanto en el promedio nacional como en algunas entidades del centro y sur del país (gráfica 1).

\section{Gráfica 1. Entidades con mayor y menor relación hombres-mujeres 2010}

(Hombres por cada 100 mujeres)

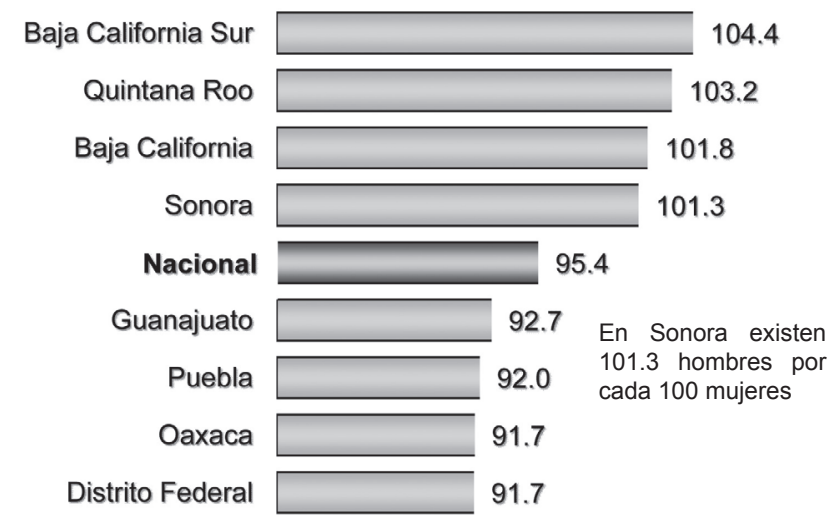

Fuente: INEGI. Censo de Población y Vivienda, 2010. Tabuladores básicos.

El número de hombres por cada 100 mujeres es diferente por grupo de edad. Generalmente nacen más niños que niñas, por lo que hasta el grupo de 20 a 29 años, hay más hombres que mujeres. Sin embargo, a partir de esta edad y debido a la mayor exposición de los hombres a la mortalidad por accidentes, agresiones y otras causas, la relación se invierte, llegando, en el grupo de 70 años y más, a contabilizar solo 89 hombres por cada 100 mujeres (gráfica 2).
Gráfica 2. Relación hombres-mujeres por grupos de edad

Sonora, 2010

(Hombres por cada 100 mujeres)

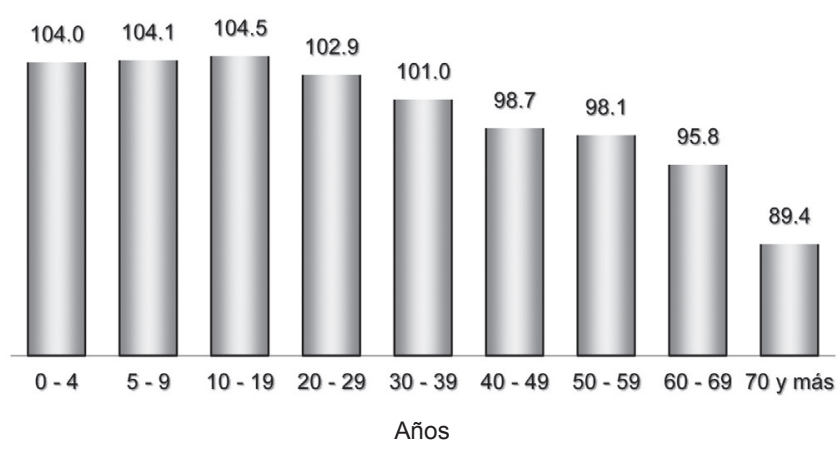

Fuente: INEGI. Censo de Población y Vivienda, 2010. Tabuladores básicos.

\section{Población de la tercera edad}

Una situación que se ha presentado en los últimos años, es la mayor importancia que, la población de 60 años y más, ha ido cobrando dentro de la población total, tanto en términos absolutos como relativos. El porcentaje de mujeres en este grupo de edad que en 1960 apenas era de 3.1\%, llegó a $5.7 \%$ en el 2010. En términos absolutos, de 12,019 mujeres, prácticamente se multiplicó por seis al llegar a 82,625. Lo anterior representa todo un reto para los sistemas de seguridad social, lo cual se agrava si pensamos que la mayor parte de las mujeres no tienen acceso a una pensión (gráfica 3 y cuadro 1$)$.

Gráfica 3. Porcentaje de población de 65 y más años respecto a la población total de cada sexo Sonora, años censales 1960, 1990 y 2010

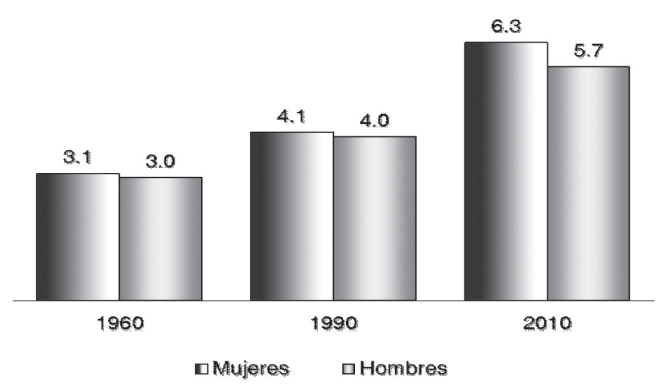

Fuente: INEGI. Censos de Población y Vivienda, 1960-2010.

Cuadro 1. Población de 65 años y más (absolutos)

\begin{tabular}{|l|c|c|}
\hline Año & Hombres & Mujeres \\
\hline 1960 & 11865 & 12019 \\
\hline 1990 & 36046 & 36762 \\
\hline 2010 & 75582 & 82625 \\
\hline
\end{tabular}

Fuente: INEGI. Censos de Población y Vivienda, 1960-2010. 


\section{Fecundidad}

Un cambio importante que la población femenina ha tenido en las últimas décadas es la menor fecundidad: de casi seis hijos que tenían las mujeres en el año de 1970, hoy día el promedio es apenas de 2.3. No obstante lo anterior, si una mujer no tuvo acceso a la escolaridad en el estado de Sonora, este promedio de 2.3 se eleva a 5.4 , aunque también influyen aspectos como el de la incorporación al mercado laboral, ya que aquellas mujeres que se encuentran insertas en el mismo, tienen en promedio menos hijos que aquellas que no lo están (gráfica 4).

\section{Gráfica 4. Promedio de hijos nacidos vivos por mujer de 12 y más años Sonora, 1970 y 2010}

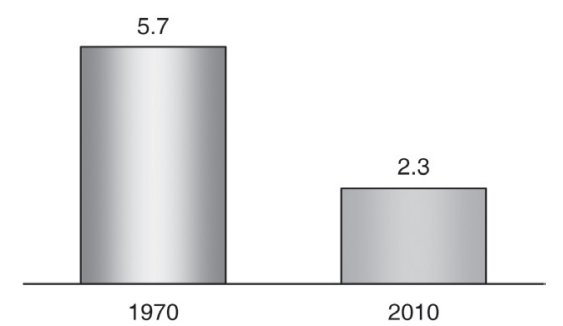

Fuente: INEGI. Censos de Población y Vivienda, 1970 y 2010.

En el tema de la fecundidad llama la atención que el porcentaje de madres menores de 20 años se ha ido incrementando en la entidad a lo largo del tiempo, y es superior al promedio nacional y al de todas las entidades de la frontera norte de nuestro país. Del total de nacimientos que se presentaron en Sonora durante el año 2012, 1 de cada 5 , es decir, el $20 \%$, fueron hijos de madres menores de 20 años. Adicionalmente, solo el $35 \%$ de las madres que tuvieron hijos en ese año estaban casadas, el $40.5 \%$ vivían en unión libre y el 19.3\% eran solteras (gráfica 5 y 6).

Gráfica 5. Nacimientos registrados

de las mujeres mejores de 20 años

Sonora, 1985 a 2012

(porcentaje)

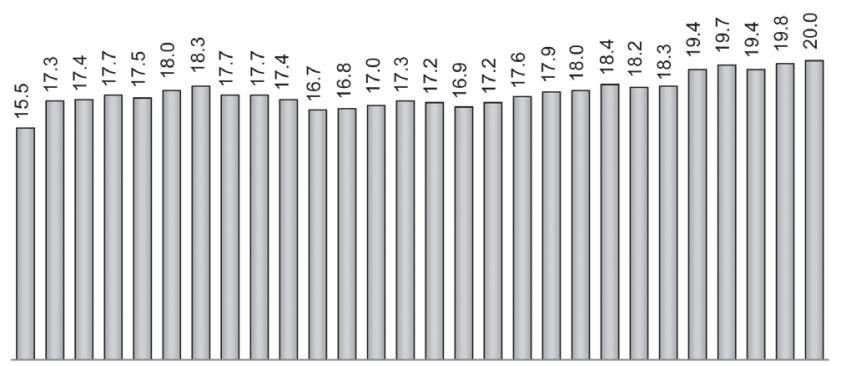

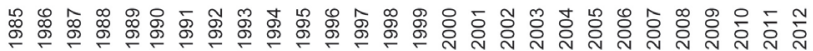

Fuente: INEGI. Estadísticas de natalidad, 1985 a 2012. Consulta interactiva de datos.
Gráfica 6. Nacimientos registrados según estado conyugal de la madre al nacimiento

Sonora, 2012

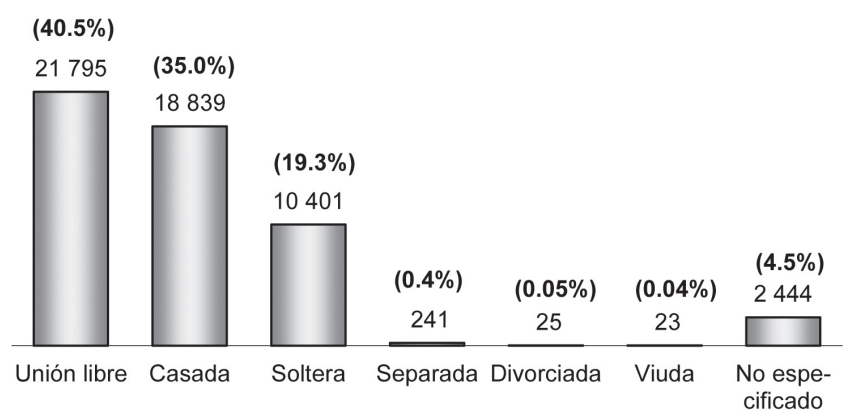

Fuente: INEGI. Estadísticas de natalidad, 2012. Consulta interactiva de datos.

\section{Aspectos conyugales}

Una característica de las mujeres que se divorcian, se separan o que quedan viudas, es que presentan una menor propensión a establecer segundas uniones con relación a los hombres. Por ello, el porcentaje de mujeres separadas, divorciadas y viudas es mucho mayor que en el caso de los hombres. Cerca de los 60 años, los hombres están unidos en el $71.4 \%$ de los casos; mientras que de las mujeres en la misma edad solo el $45.3 \%$ tiene algún tipo de unión (gráfica 7).

\section{Gráfica 7. Porcentaje de población de 12 y más años casada o unida por grupo de edad según sexo Sonora, 2010}

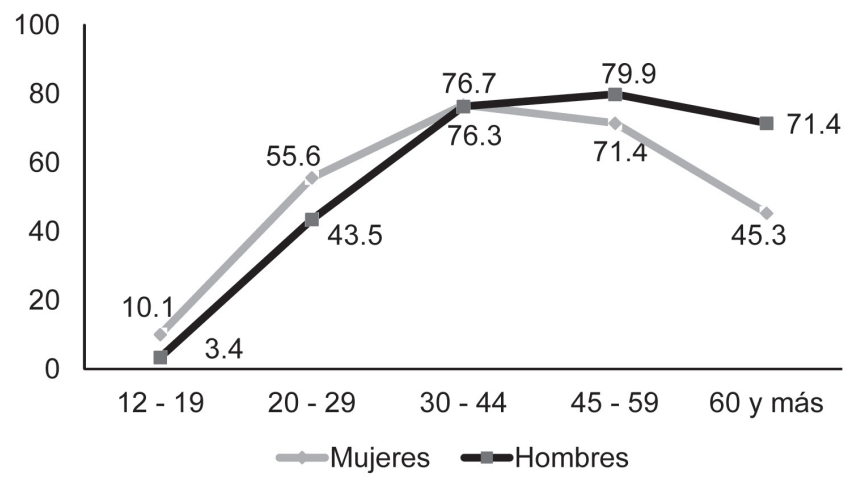

Fuente: INEGI. Censo de Población y Vivienda, 2010. Consulta interactiva de datos.

Las familias también han tenido cambios significativos. Los hogares ampliados se han incrementado en los últimos 20 años, en muchos de los casos por el apoyo y cuidado hacia los adultos mayores.

Los casos de los hogares con jefatura femenina, tanto en el ámbito nacional como en el estatal, se han incrementado significativamente en los últimos años, llegando, en el caso de Sonora, a representar el $25.7 \%$ del total. Ello significa que uno de cada cuatro hogares sonorenses tiene al frente a una mujer (gráfica 8). 
Gráfica 8. Porcentaje de hogares censales con jefatura femenina

Sonora, 2000 y 2010

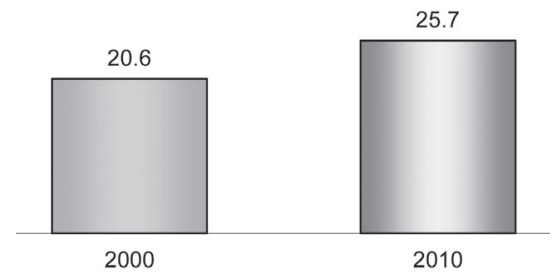

Fuente: INEGI. Censos de Población y Vivienda, 2000 y 2010. Tabuladores básicos.

\section{Educación y mercado laboral}

Otros cambios significativos en las características de la mujer sonorense son el mayor acceso a la educación y al mercado laboral, aunque hay que reconocer que ambos avances no se han dado en la misma magnitud que los hombres.

Aun cuando las mujeres sonorenses cuentan ya con un grado de escolaridad similar al de los hombres, a pesar de siglos de rezago en los cuales el acceso a las universidades para ellas estaba vedado, la participación de la mujer en el mercado laboral remunerado dista en mucho del acceso que tienen los hombres (gráfica 9 y 10).

Gráfica 9. Promedio de escolaridad de la población de 15 y más años según sexo

Sonora, 1990 y 2010

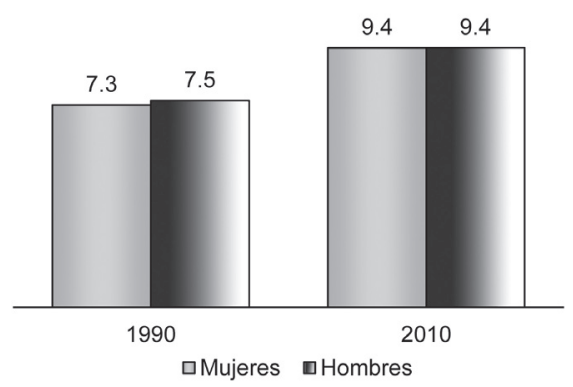

Fuente: INEGI, Encuesta Nacional de Ocupación y Empleo (ENOE), II trimestre de 2014.

Gráfica 10. Tasas específicas de participación económica de la poblaciónde 14 y más años por grupos quinquenales de edad y sexo Sonora, II trimestre de 2014

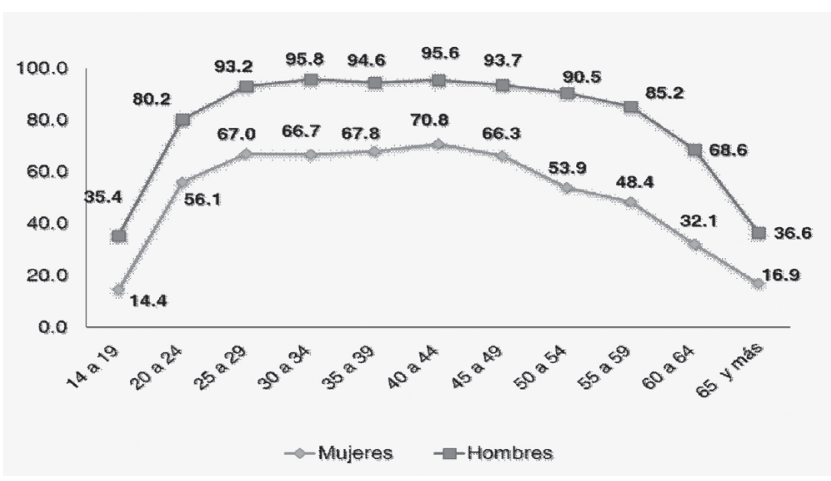

Fuente: INEGI. Censos de Población y Vivienda, 1990 y 2010.
No obstante lo anterior, Sonora es una de las entidades del país en donde la participación de la mujer en el mercado laboral es más alto, junto con Colima y Baja California Sur. Muy distantes quedan entidades del sur del país, en las cuales este indicador se encuentra más rezagado (gráfica 11).

Gráfica 11. Tasa de participación económica de la población femenina de 14 y más años en estados con mayor y menor valor Sonora, II trimestre de 2014 (porcentaje)

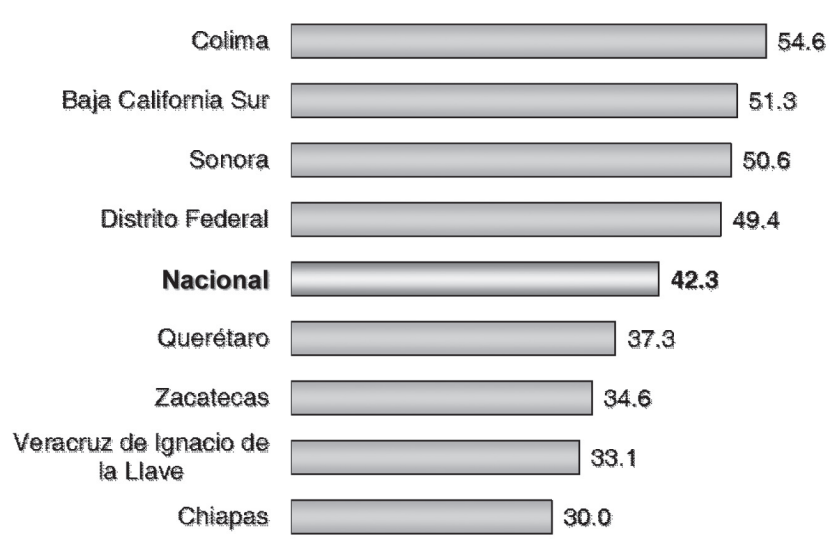

Fuente: INEGI, Encuesta Nacional de Ocupación y Empleo (ENOE), II trimestre de 2014.

La participación de la mujer en la actividad económica en Sonora se encuentra muy dirigida hacia los sectores del comercio, manufacturero (principalmente la maquiladora) y los servicios. Dentro del perfil salarial se encuentra en desventaja con los hombres, ya que en el rango de los niveles más altos (más de cinco salarios mínimos), las mujeres tienen cinco puntos porcentuales menos que los hombres y también representan menor porcentaje en la posición de empleadoras (gráfica 12, 13 y 14).

Gráfica 12. Distribución porcentual de la población ocupada por sector de actividad económica y sexo Sonora, II trimestre de 2014

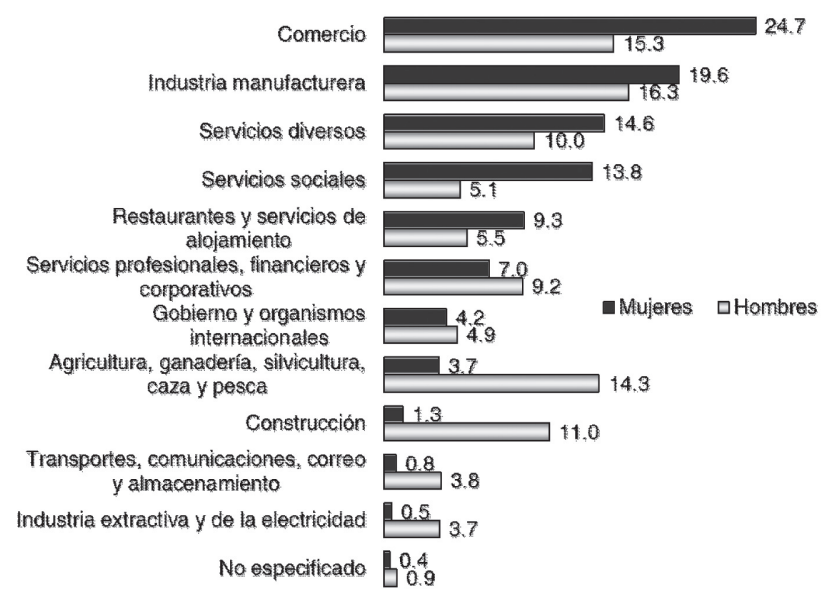

Fuente: INEGI, Encuesta Nacional de Ocupación y Empleo (ENOE), II trimestre de 2014. 
Gráfica 13. Distribución porcentual de la población ocupada según nivel de ingreso por trabajo ${ }^{\mathrm{a} /}$ y sexo

Sonora, II trimestre de 2014

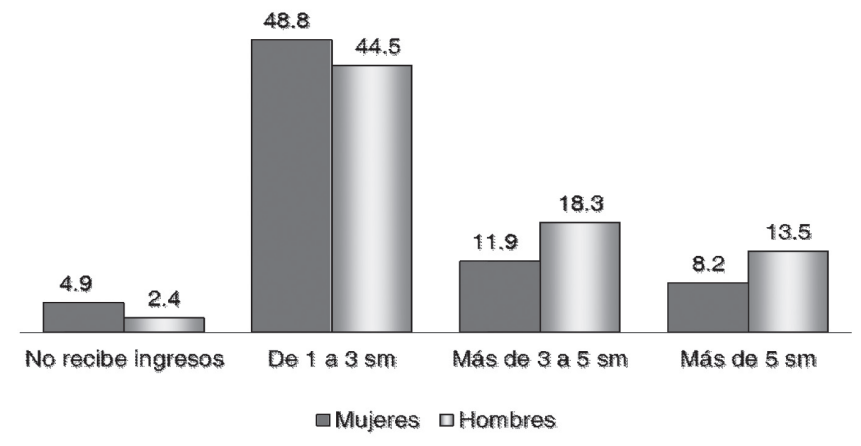

Nota: la suma de los datos no da el $100 \%$ debido a que no se incluye a quienes perciben hasta un salario mínimo, ni el no especificado.

a/ se expresa en salario mínimo (sm)

Fuente: INEGI, Encuesta Nacional de Ocupación y Empleo (ENOE), II trimestre de 2014

Gráfica 14. Distribución porcentual de la población ocupada según posición en la ocupación y sexo

Sonora, II trimestre de 2014

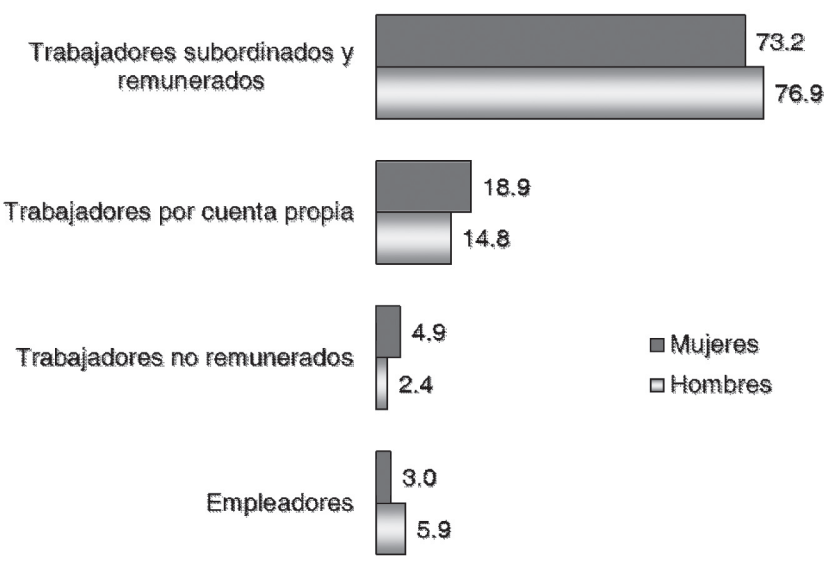

Fuente: INEGI, Encuesta Nacional de Ocupación y Empleo (ENOE), II trimestre de 2014.

\section{Salud}

En el tema de la salud, las mujeres siempre han mantenido una esperanza de vida mayor que la de los hombres. En promedio, una mujer sonorense vive 5 años más que un hombre. Esto tiene que ver con el hecho de que la sobremortalidad masculina es mayor, incluso tratándose de niños de edad temprana. Con el paso del tiempo, los hombres tienen mayor exposición a fallecimientos por accidentes, agresiones e infartos, entre otras causas, que llevan a que en el grupo de 25 a 34 años, un hombre tenga un índice de sobremortalidad de 325.1 (gráfica 15).

En cuanto a las causa de muerte, en el caso de las mujeres, las más relevantes son las enfermedades del corazón, los tumores malignos (dentro de los cuales el cáncer de mama ha crecido en importancia en los últimos años) y la diabetes mellitus, que amenaza con convertirse dentro de pronto en la segunda causa de muerte en las mujeres, rebasando a todos los tipos de cáncer (gráfica 16).
Gráfica 15. Índice de sobremortalidad masculina por grupos de edad Sonora, 2012

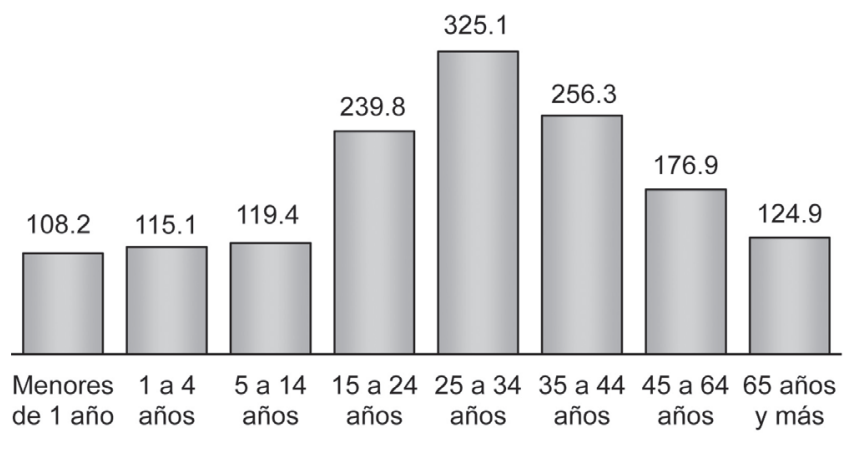

Fuente: INEGI. Estadísticas de mortalidad, 2011. Consulta interactiva de datos.

Gráfica 16. Principales causas de muerte en la poblaciónpor cada sexo

Sonora, 2012

(porcentaje)

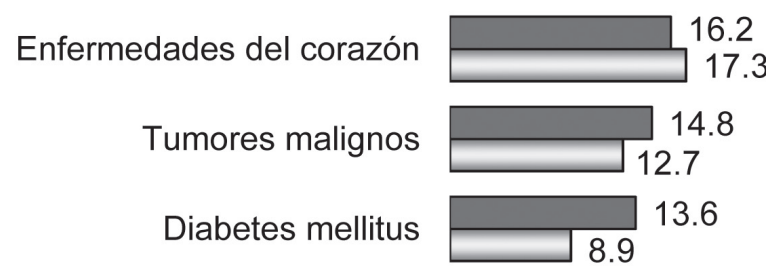

Enfermedades $\square .4$ cerebrovasculares $\square 4.1$

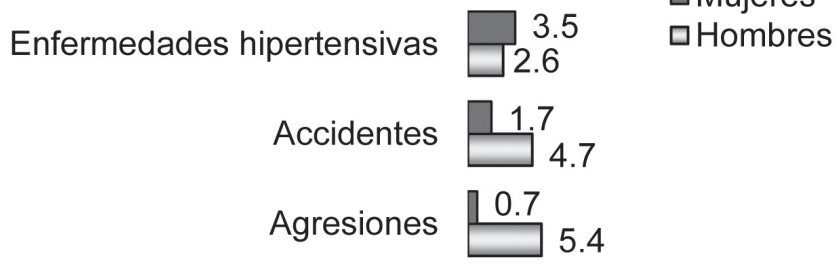

Fuente: INEGI. Estadísticas de mortalidad, 2012. Consulta interactiva de datos.

\section{Trabajo doméstico}

Un aspecto relevante que ha presentado cambios en los últimos años es la división del trabajo doméstico. Para el año 2009 poco más del $80 \%$ de este tipo de actividad en nuestro país lo realizaban las mujeres. Se observa una reducción respecto del año 2002, en el cual la carga para las mujeres era casi del 85\%. Lo anterior implica que cada vez más, los hombres asumen este tipo de tareas que histórica y culturalmente han sido asignadas a las mujeres (gráfica 17).

La mayor carga del trabajo doméstico para las mujeres se manifiesta en todas las edades y situaciones conyugales. En las mujeres solteras, la asignación de tareas en los hogares es el doble que en el caso de los hombres solteros. Peor aún, cuando una mujer se casa, aumenta su jornada semanal en 40 horas adicionales, a las casi 28 que ya sostenía mientras era soltera (gráfica 18).

\section{Savĩa}


Gráfica 17. Promedio de horas a la semana destinadas al trabajo doméstico según sexo

Nacional, 2002 y 2009

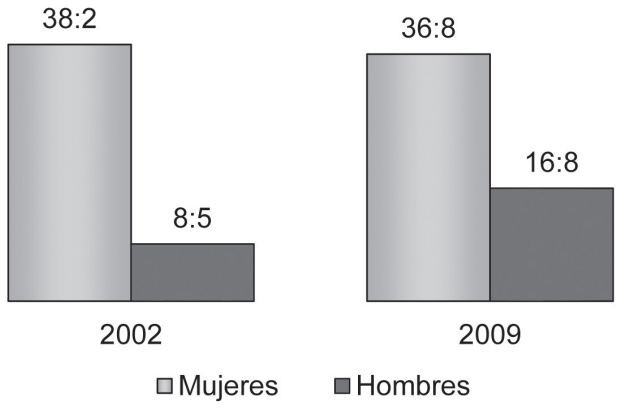

Nota: los promedios de horas se presentan en unidades de horas y minutos Fuente: INEGI. Encuesta Nacional sobre Uso del Tiempo (ENUT), 2002 y 2009

Gráfica 18. Promedio de horas a la semana destinadas al trabajo doméstico y cuidado de niños (as) y apoyo a otros miembros del hogar por situación conyugal de la población según sexo

Nacional, 2009

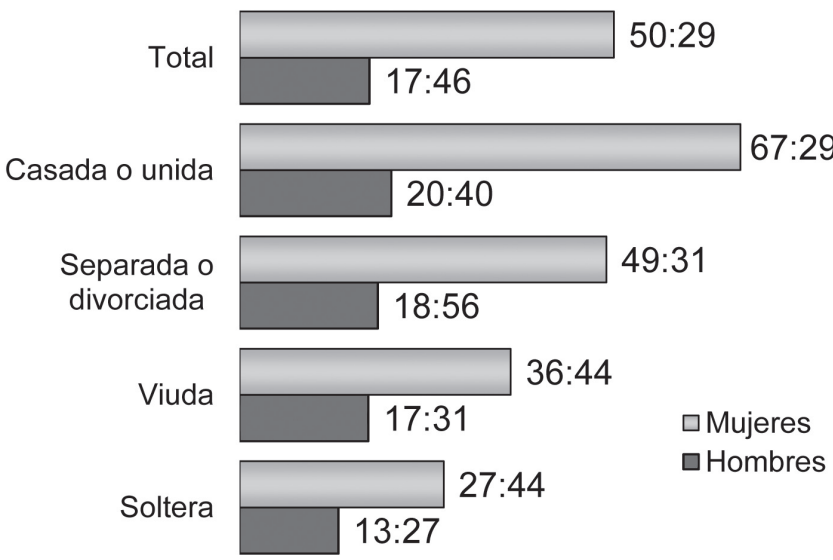

Nota: los promedios de horas se presentan en unidades de horas y minutos Fuente: INEGI. Encuesta Nacional sobre Uso del Tiempo (ENUT), 2009.

\section{Violencia hacia las mujeres}

Por último, está el tema de la violencia, que si bien es un fenómeno que lamentablemente han padecido las mujeres desde tiempos remotos, es en fechas recientes cuando se inicia la medición estadística sobre este hecho.

De acuerdo con diversas encuestas realizadas, la violencia que sufre una mujer por parte de su pareja se clasifica en emocional, económica, física y sexual. Si las agrupamos en un solo indicador, y nos referimos a la violencia reciente (en el último año anterior a las encuestas), en Sonora, prácticamente cuatro de cada diez mujeres sufrió violencia por parte de su pareja. Esto ubica al estado en un poco grato cuarto lugar en el contexto nacional. Se destaca además que la violencia es padecida en mayor grado por las mujeres más jóvenes y las que se encuentran incorporadas al mercado laboral (gráfica 19, 20 y 21).
Gráfica 19. Porcentaje de mujeres casadas o unidas violentadas por su pareja en los últimos 12 meses por entidad federativa. 2011

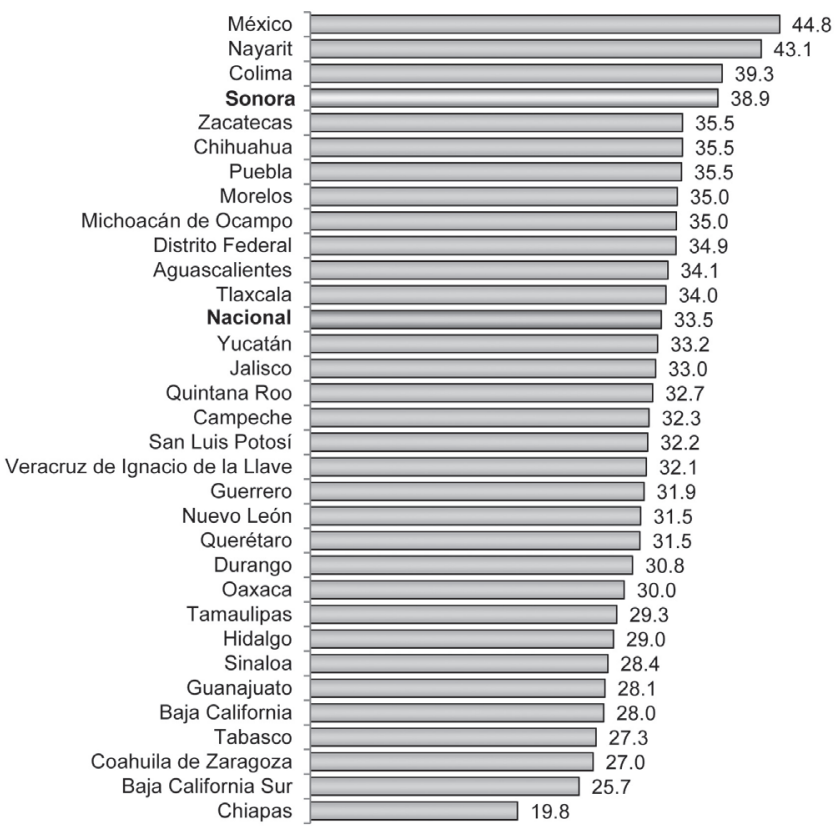

Fuente: INEGI. Encuesta Nacional sobre la Dinámica de las Relaciones en los Hogares, 2011.

\section{Gráfica 20. Porcentaje de mujeres casadas o unidas violentadas por su pareja en los últimos 12 meses según grupos de edad Nacional y Sonora. 2011}

2011

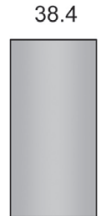

15 a 29

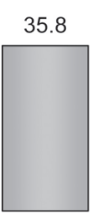

30 a 44 Nacional
51.1

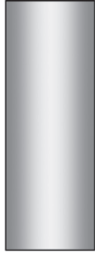

15 a 29

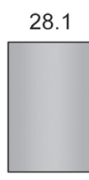

45 y más

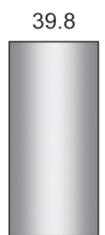

30 a 44 Sonora
31.8

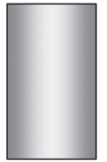

45 y más
Fuente: INEGI. Encuesta Nacional sobre la Dinámica de las Relaciones en los Hogares, 2011.

Gráfica 21. Porcentaje de mujeres casadas o unidas violentadas por su pareja en los últimos 12 meses, según condición de actividad Nacional y Sonora. 2011
2011

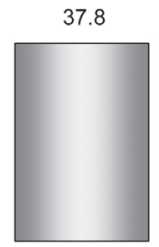

Económicamente activas

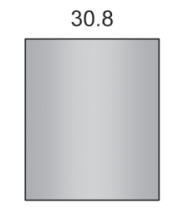

oconómicamente activas
42.7

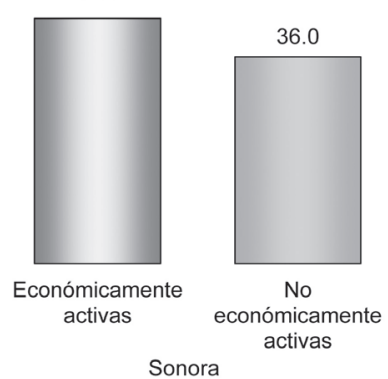

Fuente: INEGI. Encuesta Nacional sobre la Dinámica de las Relaciones en los Hogares, 2011. 
Pero no solo las mujeres que viven con su pareja están expuestas a sufrir violencia por parte de ella. Las mujeres solteras muestran niveles altos de violencia que viven por parte de sus novios. Desafortunadamente, Sonora ocupa lo segunda posición en el contexto nacional con relación a la violencia en el noviazgo, ya que prácticamente la mitad de las mujeres viven o han vivido esta situación por parte de sus parejas (gráfica 22).

Gráfica 22. Porcentaje de mujeres solteras violentadas por su novio o exnovio a lo largo de su relación por entidad federativa 2011

2011

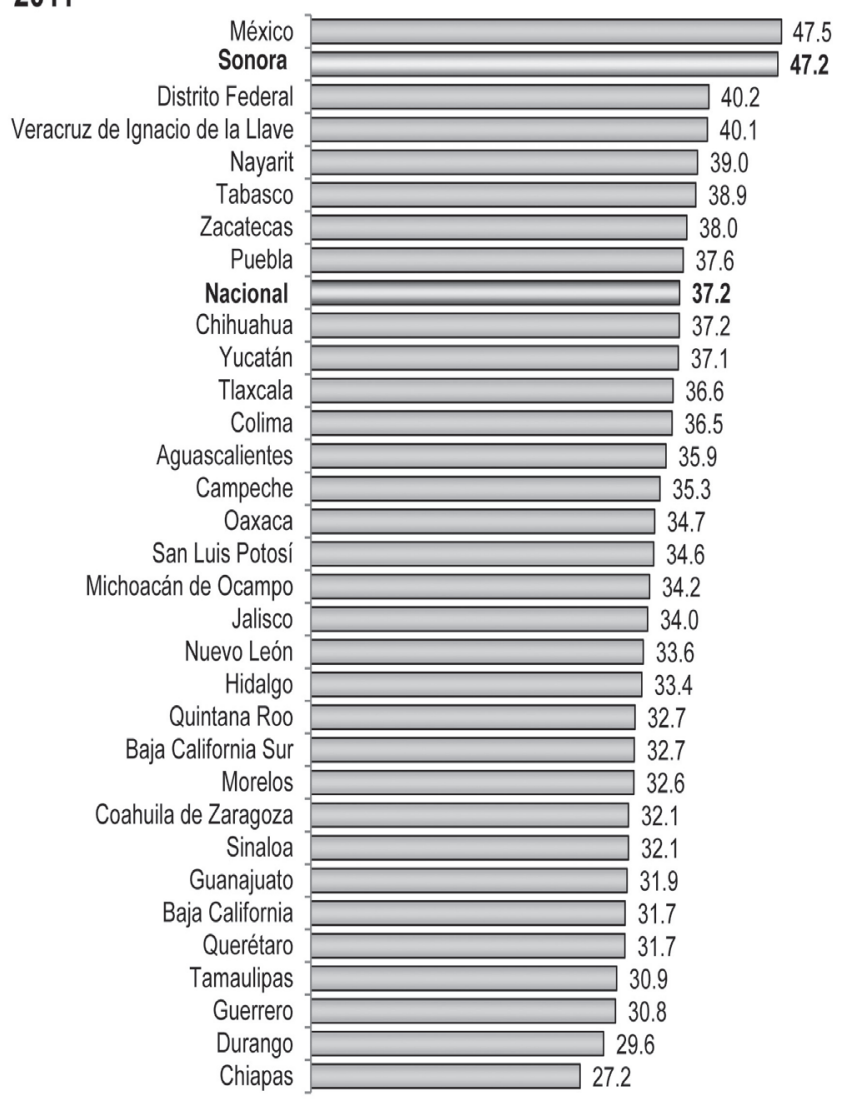

Fuente: INEGI. Encuesta Nacional sobre la Dinámica de las Relaciones en los Hogares, 2011.

Adicionalmente, las mujeres también están expuestas a sufrir otros tipos de violencia en los ámbitos comunitarios, escolar y laboral. Particularmente, en este último aspecto, el $20.8 \%$ de las mujeres, es decir, una de cada cinco, han sufrido violencia laboral; principalmente la discriminación, que se manifiesta por hechos como tener menores oportunidades para ascender, solicitud de la constancia de no gravidez como requisito para la contratación o menores sueldos (gráfica 23).
Gráfica 23. Porcentaje de mujeres ocupadas violentadas en el ámbito laboral

Nacional y Sonora. 2011

\section{1}

22.6

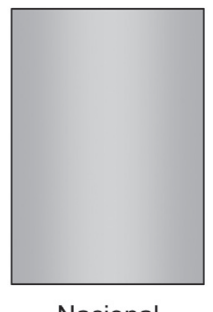

Nacional
20.8

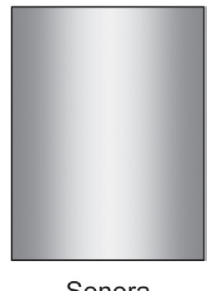

Fuente: INEGI. Encuesta Nacional sobre la Dinámica de las Relaciones en los Hogares, 2011.

\section{Comentarios finales}

La disponibilidad actual de información estadística nos permite analizar los avances que en materia de género se han logrado hasta el momento, pero también, permite dimensionar fenómenos que hasta hoy se ubican como temas pendientes en las agendas de los gobiernos y sociedad: la violencia de género, la discriminación laboral, la incidencia de cáncer de mama y diabetes como causas de muerte, entre otros.

Se espera que el uso de esta información sea aún más intensiva por los diversos sectores de la sociedad para evaluar y diseñar las políticas públicas encaminadas a lograr una mayor equidad.

\section{Bibliografía}

Instituto Nacional de Estadística y Geografía (INEGI). Censo de Población y Vivienda 2010. Tabulados básicos y Consulta interactiva de datos. Recuperado de: http://www. inegi.org.mx/est/contenidos/proyectos/ccpv/cpv2010/

Instituto Nacional de Estadística y Geografía (INEGI). Encuesta Nacional de Uso del Tiempo 2009. Base de datos. Recuperado de: http://www.inegi.org.mx/est/contenidos/ Proyectos/Encuestas/Hogares/especiales/enut/enut2009/ default.aspx

Instituto Nacional de Estadística y Geografía (INEGI). Estadísticas de Natalidad. Consulta interactiva de datos. Recuperado de: http://www.inegi.org.mx/sistemas/ olap/Proyectos/bd/continuas/natalidad/nacimientos. asp?s=est\&c=23699\&proy=nat_nac

Instituto Nacional de Estadística y Geografía (INEGI). Estadísticas de Mortalidad. Consulta interactiva de datos.

Instituto Nacional de Estadística y Geografía (INEGI). Encuesta Nacional de Ocupación y Empleo. II trimestre de 2014. Indicadores estratégicos.

Instituto Nacional de Estadística y Geografía (INEGI). Encuesta Nacional de la Dinámica de las Relaciones en los Hogares 2011. Base de datos. 

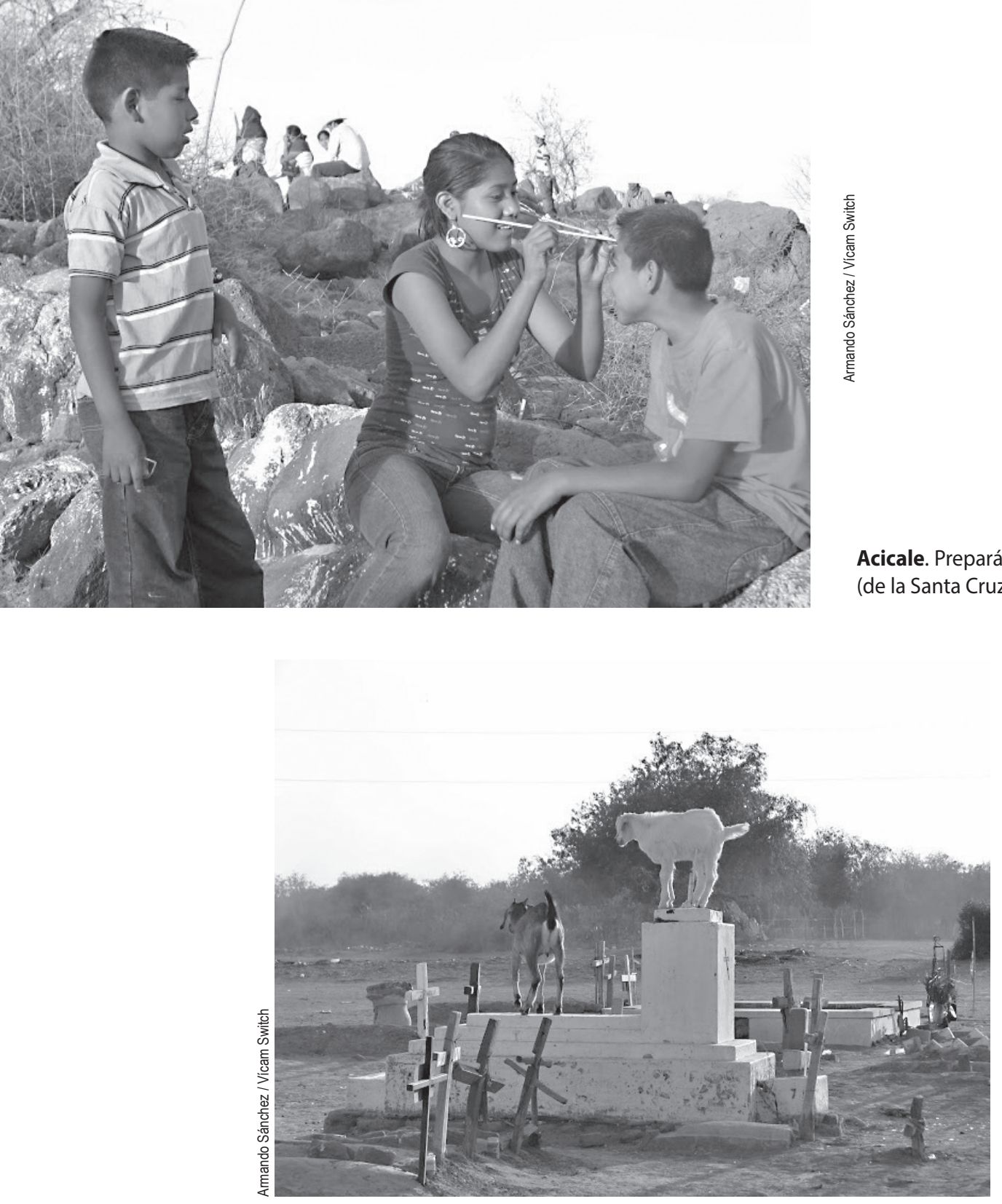

Los Chivos... Pero no se confíe porque por la tarde estos congéneres de muchos maridos viqueños suelen divertirse $y$ comer flores, si las hay (A. Valenzuela).

Orgullo motorizado. Haiga sido como haiga sido, pero tenemos carro (A. Valenzuela).

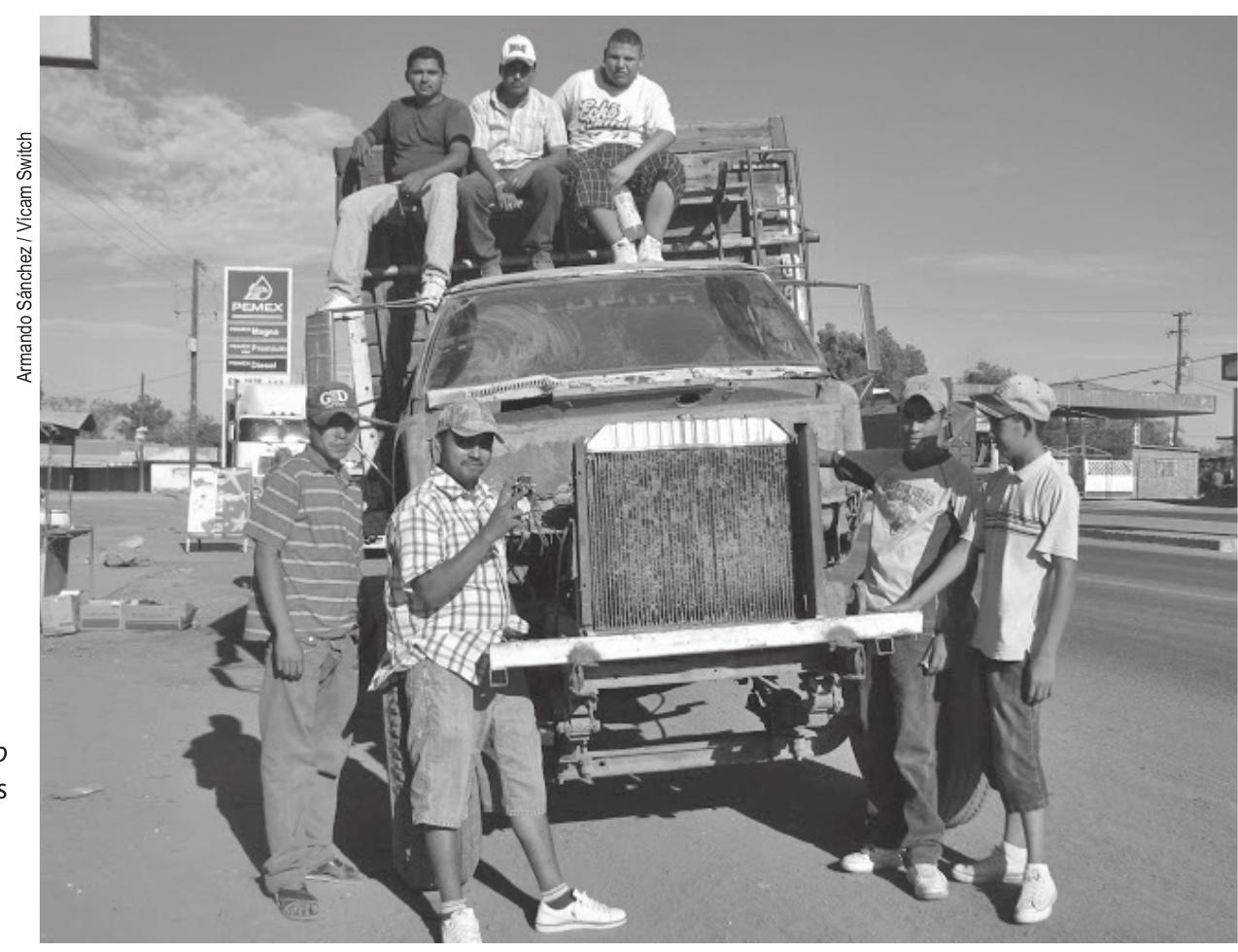

